\title{
Les habits neufs du communautarisme libanais
}

\section{Elisabeth Picard}

\section{(2) OpenEdition}

1 Journals

\section{Édition électronique}

URL : http://journals.openedition.org/conflits/515

DOI : 10.4000/conflits.515

ISSN : $1777-5345$

Éditeur :

CCLS - Centre d'études sur les conflits lilberté et sécurité, L'Harmattan

\section{Édition imprimée}

Date de publication : 15 octobre 1994

ISSN : 1157-996X

Référence électronique

Elisabeth Picard, "Les habits neufs du communautarisme libanais », Cultures \& Conflits [En ligne], 15-16 | automne-hiver 1994, mis en ligne le 15 mars 2006, consulté le 30 mars 2021. URL : http:// journals.openedition.org/conflits/515; DOI : https://doi.org/10.4000/conflits.515

Ce document a été généré automatiquement le 30 mars 2021.

Creative Commons License 


\title{
Les habits neufs du communautarisme libanais
}

\author{
Elisabeth Picard
}

On surprendrait vivement les leaders politiques libanais de l'avant-guerre, les Pierre Gemayel, les Rachid Karamé, les Camille Chamoun et autres Kamal Junblat - Allah yarhamôn - en leur apprenant que le " confessionnalisme ", la structuration de la société en communautés religieuses régies par leur droit privé spécifique, n'est plus guère contesté aujourd'hui à Beyrouth. Et plus encore en constatant que le " communautarisme politique ", soit l'inscription de la division communautaire dans le système constitutionnel du pays, est sorti renforcé de quinze années de guerre. Avec l'avenir des Palestiniens et la satisfaction des ambitions de son voisin syrien, la question de la levée de l'emprise communautaire sur l'Etat avait été un enjeu majeur des années 1970, un objet de discorde entre ces leaders, et une cause de l'embrasement du Liban. Pour régler la question communautaire et faire avancer la laïcisation du système politique, propositions et contre-propositions s'étaient succédées au long de la guerre: programme intérimaire de réforme politique du Mouvement National (août 1975), document constitutionnel du Président Frangié (février 1976), propositions du Front Libanais (janvier 1977), quatorze points du Président Sarkis (mars 1980), document du Haut comité druze (mai 1983), projet du mouvement Amal (novembre 1983), conclusions de la réunion de Lausanne (mars 1984), accord intermilicien " de Damas " (décembre 1985), projet du Président Gemayel (mars 1987), projet de réforme de Rafic Hariri (novembre 1987) 1, sans oublier les propositions étrangères américaines et syriennes en particulier. Or, depuis l'adoption de la nouvelle Loi constitutionnelle du 21 septembre 1990², les critiques envers le communautarisme politique se sont considérablement atténuées, et les demandes de laïcisation du droit personnel ou de séparation du religieux et du politique se sont faites rares. Lorsqu'elles sont exprimées, elles sont parfois accueillies avec mépris. Ainsi l'ancien président du Conseil, Salim el-Hoss, adversaire obstiné du communautarisme politique, est-il soupçonné de " [faire] du confessionnalisme sommaire " 3. Souvent, elles sont contrées avec l'aplomb de ceux qui se savent porteurs d'une opinion majoritaire et défenseurs d'un système établi. Dans le Liban d'après-guerre, le communautarisme va de soi ; il 
reflète l'état de la société; il assure le fonctionnement de l'Etat. Le remettre en cause serait dangereux pour l'équilibre du pays, ou tout simplement impossible au vu du rapport des forces sociales et politiques. Ce constat nous incite à nous interroger à propos de la nouvelle République libanaise, sur la nature des arrangements intercommunautaires qui la fondent, sur les sources de sa stabilité et sur les menaces que ferait peser sur ces populations la sécularisation du système politique. Mais avant cela, il nous faudra examiner les causes et les circonstances du changement radical que constitue le triomphe du discours communautaire dans le Liban de l'après-guerre. Ensuite, seulement, nous remettrons en cause la relation entre Etat et communautés, dans une perspective dynamique. Sans nous arc-bouter rigidement sur le paradigme de l'Etat-nation, nous soulèverons quelques questions à propos de la légitimité de l'Etat libanais de la Deuxième république, et de sa fonction de régulation de l'économie nationale. Nous espérons montrer que, même s'il se pose différemment, le problème des réformes constitutionnelles est toujours d'actualité.

2 La réhabilitation de l'ethnicité A la veille de la guerre du Liban, à l'époque dite " moderne ", la structuration communautaire de la société libanaise était souvent considérée comme un phénomène honteux, et l'organisation communautaire de l'Etat comme un regrettable vestige des époques ottomane et mandataire. Dans un contexte de croissance économique et de changement social rapide, les comportements laïques, c'est-à-dire détachés du lien communautaire, s'affichaient. Ils étaient particulièrement observables dans deux domaines: la multiplication des mariages mixtes, rendue possible par des conversions de complaisance ou par le recours à une juridiction laïque (en général, à Chypre ou en France) ; et l'extension des zones d'habitat mixte, soit en milieu prolétaire dans les nouvelles banlieues industrielles, soit au contraire dans les luxueux quartiers de Hamra, Raouché et Ramlet el-Baïda, à Beyrouth ouest. Cette évolution des comportements s'appuyait sur un changement des discours et des postures. Pendant des décennies, les relations intercommunautaires avaient été gérées par des codes de dissimulation des identités et des antagonismes. Or, en même temps que s'estompait la mémoire des coutumes nécessaires à l'évitement, la " pudeur " ${ }^{4}$ des communautés cédait peu à peu la place à l'indifférence et une culture urbaine standardisée se développait. De plus, les idéologies laïcisantes gagnaient en influence, véhiculées par la résistance palestinienne, par les échos de mai 1968 dans les milieux étudiants et plus encore par l'adoption de modèles occidentaux. Le succès de l'économie marchande et du réseau bancaire libanais ne connaissait pas de frontière communautaire ; la crise (hausse de prix, crise du logement) qui frappait les couches populaires, non plus. A la veille de la guerre, sayyed Musa Sadr mobilisait les Chiites sous la bannière du Mouvement (en principe transcommunautaire) des Déshérités. Lorsque la guerre s'est déclenchée, le Front Libanais qui s'opposait aux Palestiniens a été perçu comme conservateur (ce qu'il était) plutôt que comme chrétien (ce qu'il était plus nettement encore). La révision induite par la guerre a été radicale. Les identités culturelles collectives sont aujourd'hui reconnues. Elles sont même affichées, et les particularismes revendiqués. Etrange inversion du discours dominant, remarque l'historien Nadim Shehadi ${ }^{5}$, qui non seulement lève un tabou, mais érige la socialisation communautaire en norme. L'insécurité, la dispersion des familles, le passage des générations, la perte des repères socio-économiques en période d'anomie et, bien sûr, la paralysie, puis l'éclatement de l'appareil d'Etat, tous ces facteurs ont contribué à revaloriser la communauté religieuse comme marqueur ultime des identités ${ }^{6}$. La différence communautaire est devenue le principe central de l'explication politique et 
le moteur premier de la mobilisation politique: les Chiites sont soupçonnés d'être favorables à un (hypothétique) régime islamiste; les Chrétiens sont globalement assimilés à l'opposition au gouvernement de Rafic Hariri. Le statut des Chrétiens dans un Orient majoritairement musulman devient l'enjeu politique majeur. Les hiérarchies religieuses se substituent aux élites laïques absentes ou délégitimées. La guerre a été la cause principale de la modification radicale des représentations sociales qui servent de matrices aux identités communautaires renforcées. Mais le contexte mondial de la post-modernité n'est pas moins important: un contexte dans lequel la guerre des cultures, ou le " clash of civilizations ", s'est substitué au conflit bipolaire comme système d'explication du monde. La revendication identitaire - communautaire, ethnique, nationalitaire ou nationale - s'est partout exacerbée. Depuis la fin des années 1980, les guerres communautaires se sont multipliées dans l'aire méditerranéenne et moyen-orientale: Caucase, Balkans et Corne de l'Afrique. Et, s'il fallait une preuve supplémentaire, le Liban, naguère synonyme d'Etat paisible et démocratique, est devenu la référence à un processus d'éclatement de l'Etat et de conflit communautaire : la " libanisation ". Autant de raisons qui se conjuguent pour penser et agir le politique en termes communautaires.

3 Un communautarisme renforcé Le " document d'entente nationale " adopté à Taëf par 58 des 70 députés libanais encore vivants en octobre 1989, et entériné par la nouvelle Loi constitutionnelle, a confirmé le principe du communautarisme politique en maintenant le fameux article 95 qui stipule que " dans la période transitoire [qui dure depuis 1926]... les communautés seront équitablement représentées dans la fonction du ministère " 7 . Il a fait plus. Car au Pacte national de 1943, formule implicite et non écrite de coexistence communautaire, il a désormais substitué la formule écrite de " pacte de vie en commun ", condition exigée à la légalité du pouvoir ${ }^{8}$. La recherche de l'accommodement intercommunautaire s'opère au niveau de l'exécutif, partagé par le Conseil des ministres et le Président, au niveau du Législatif, le Parlement qui dispose de pouvoirs sensiblement accrus, et jusque dans les hauts rangs de l'administration dont les 120 principaux fonctionnaires sont choisis de concert par les trois Présidents (de la République, du Conseil et de l'Assemblée). A ce choix, nous pouvons avancer trois modes d'explication, qui se complètent plus qu'ils ne s'opposent. Le premier consiste à reconnaître que le " document " de Taëf ne faisait qu'entériner une situation de fait. Rompant avec les multiples et vaines tentatives de réforme qui avaient rythmé la guerre, il prenait acte de la présence (mais non de la victoire) partout sur le terrain, de forces politiques et militaires à base communautaire, en même temps que du silence, ou de la disparition, des forces transcommunautaires de la société civile. Après tant de désastres idéologiques et de coûteux fantasmes, seules comptaient désormais les réalités tangibles : les forces de facto (les milices) et les fa'âliyât ${ }^{9}$, et c'était avec elles qu'il fallait reconstruire le pays. Puisque la communauté s'était révélée l'unité résiliente de la structure sociale libanaise, la plus immédiatement opérationnelle aussi, le système constitutionnel reflétait cette structure et organisait le fonctionnement de ses éléments. Le choix de Taëf avait aussi une autre explication: l'identité des signataires et des promoteurs du document d'entente. Ce sont les députés, élus de 1972 et représentants par excellence des communautés, qui ont discuté et adopté ce texte. Convoqués autoritairement à Taëf, réinstallés par miracle en position de décideurs alors qu'ils avaient perdu leur crédit populaire en même temps que leur capacité à peser sur le destin de l'Etat, comment n'auraient-ils pas saisi cette occasion de recouvrer légitimité et pouvoir? Pour mettre fin à la division du Liban en quasi Etats 
dominés par les milices, ils se sont entendus au moins pour restaurer le système de représentation et d'accommodement communautaire qui les avait portés au pouvoir en 1972. Mais ce " miracle " était surtout le fait d'un deus ex machina, ou plutôt de deux puissances régionales. L'Arabie Saoudite, d'abord, favorable, dans toute la région arabe, à des régimes politiques fondés sur une foi religieuse, ou taillés à la mesure des communautés de croyants. Pour les dirigeants saoudiens, un régime dominé par une communauté chrétienne, comme le régime libanais l'était par les Maronites avant la guerre, est préférable à un régime sécularisé ; un Etat communautaire dont l'exécutif est dominé par un président du Conseil musulman sunnite, comme l'est le régime libanais mis en place à Taëf, comble leurs voeux. La Syrie, ensuite, trouvait dans la formule de Taëf la consécration d'un maintien illimité de son contrôle militaire sur le Liban. Car, tout en approuvant la restauration du système communautaire et en marquant sa sollicitude pour les chefs de communautés, religieux et politiques, elle obtenait l'insertion, dans le chapitre des réformes, d'une exigence ferme d'abolition du communautarisme politique, abolition à laquelle elle conditionnait le retrait de ses forces ${ }^{10}$. En somme, la pérennisation du communautarisme assurait la pérennisation de son occupation.

4 Un rempart contre les totalitarismes Conservatisme, force d'inertie et instrumentalisation d'une stratégie par des acteurs sont des explications convaincantes $\mathrm{du}$ renforcement $\mathrm{du}$ communautarisme politique après la guerre du Liban. Ces explications doivent pourtant être complétées par l'examen de thèses abondamment débattues à Beyrouth, selon lesquelles l'adoption d'un mode de représentation laïque, basé sur une majorité démographique, comporte un sérieux risque de dérive totalitaire. La première thèse consiste à opposer les voies constitutionnelles choisies par différents Etats successeurs de l'Empire Ottoman au Moyen-Orient, le Liban d'une part, les autres Etats arabes de l'autre, après la décolonisation. Alors que le premier faisait accéder les communautés à la représentation politique, reprenant en le transformant le système des millet ${ }^{11}$, les autres récusaient ce mode d'organisation " traditionnel " (de même que le tribalisme). Pour accéder à la modernité, ils choisissaient théoriquement la voie de la sécularisation et de l'intégration nationale, celle dans laquelle les citoyens égaux en droits et en devoirs ont l'Etat pour interlocuteur. En principe, donc, la majorité politique de ces pays est dessinée par la seule loi du nombre. En pratique, leur sécularisation est incomplète, puisque l'islam ou la sharî'a figurent, à un titre ou un autre, dans toutes les constitutions du monde arabe. Surtout, la plupart de ces systèmes, loin d'assurer une compétition équitable, favorisent la domination d'une communauté (un segment ethnique ou religieux de la population) sur les autres ${ }^{12}$. Ainsi, la soi-disant sécularisation d'Etats comme la Syrie ou l'Iraq masque un système de préférence et d'exclusion communautaire plus virulent que le communautarisme institutionnalisé, parce qu'il échappe à la régulation constitutionnelle. En dénonçant le caractère dictatorial de régimes " modernes " et sécularisés, en proclamant même que le Liban est le seul Etat de la région qui a échappé au totalitarisme, les défenseurs du communautarisme politique mettent l'accent sur un autre problème: celui du déficit démocratique. Or, ce n'est pas parce qu'ils sont laïques que ces régimes arabes sont dictatoriaux, mais plutôt parce qu'ils ne le sont qu'en façade et, qu'au fond, ils sont farouchement communautaristes. Ils ont dévoyé le principe laïque de la séparation du religieux et du politique en identifiant l'Etat à une seule communauté; ils ont transformé le principe démocratique " un homme, un vote " en un plébiscite sans choix ; ils ont " dé-politisé " les opposants au système en les excluant d'une nation pour 
le moins hypothétique. Mais, pour aigu qu'il soit dans ces régimes patrimoniaux et militarisés, le problème de la démocratie ne se pose pas moins en régime communautaire. La différence confessionnelle induit l'inégalité face à l'Etat, comme c'était le cas dans le Liban avant la guerre. La communauté risque, elle aussi, d'imposer à l'individu sa propre forme d'enfermement totalitaire, celle qui s'est développée durant la guerre avec le système des milices ${ }^{13}$. La seconde thèse conduit à soupçonner, derrière les demandes de laïcisation et d'adoption de la majorité du nombre, l'ambition dictatoriale d'une majorité sociologique, celle d'une communauté, qui chercherait à imposer à l'ensemble de la population son système de sens et de droit. La communauté visée est la communauté chiite. Toute polémique mise à part et toute supputation sur les chiffres écartée, celle-ci est unanimement reconnue comme la plus nombreuse, et donc comme sociologiquement (mais non démographiquement; elle représente moins de 50\% de la population) majoritaire. C'est un fait que, dans le Liban des années 1990, la demande de déconfessionnalisation est formulée surtout par deux ténors: Georges Corm, d'une part, parce qu'il est un adversaire implacable des dictatures miliciennes ${ }^{14}$. Et, d'autre part, le Hizb Allah, assuré qu'il est de son hégémonie en milieu chiite après sa victoire militaire sur Amal en 1990 et son succès aux législatives de l'été $1992^{15}$. Certes, le projet politique du Hizb est fluctuant. Il varie en fonction du rapport de forces au sein du Majlis el-shûra, son conseil directeur, de ses relations avec le gouvernement, et surtout d'une stratégie inscrite dans le triangle étatique Syrie-IranIsraël. N'empêche que les Islamistes se caractérisent partout par leur demande d'application de la sharî'a, y compris l'application d'un statut spécifique aux Gens du Livre. Par la loi du nombre, le Liban " laïque " risquerait de se voir transformé en République islamique. Il n'y a guère de réponse satisfaisante à cette inquiétude, même si les Libanais peuvent au moins se réjouir de ne pas être confrontés un jour à l'alternative régime islamique/régime militaire, comme ce pourrait être le cas de leur voisin syrien. Au moins la réalité sociologique du pluralisme communautaire, telle que nous l'observons à Beyrouth ouest, dans la dhahiyya (sa banlieue sud) et dans la région rurale de Nabatiyeh permet-elle de relativiser l'adhésion des Libanais chiites au projet politique du Hizb Allah. D'une part, cette population touchée par une urbanisation et une modernisation rapide connaît un processus d'individuation, particulièrement repérable à travers un investissement spectaculaire dans le domaine de la scolarisation et de la formation. D'autre part, la communauté chiite, comme les autres communautés libanaises, loin d'être monolithique, est marquée par des diversités régionales (Beqaa ou Liban sud), religieuses (tenants et opposants du wilâyat al-faqîh ; fidèles d'un marja' ou d'un autre ; supporters ou adversaires du Conseil supérieur chiite; partisans d'une Ashûra' quiétiste ou politisée, etc.), des diversités politiques (gauchistes, communistes et nationalistes syriens du PPS, contre les clans et les grandes familles, mais aussi contre les nouvelles élites légalistes de Amal) et aussi par une pluralité des centres de pouvoir économique (avec une influence décisive de l'émigration mais aussi des banquiers engagés dans des opérations transcommunautaires). Avant même les membres des autres communautés, les Chiites pourraient dénoncer la tentation totalitaire du projet islamiste et réclamer, eux aussi, une laïcisation authentique ${ }^{16}$.

5 Consentement ou confrontation? Plutôt que de parier sur l'avenir, et instruits du contexte régional dont dépend leur propre pays, une majorité de Libanais souhaite donc conserver le régime du communautarisme politique, en adhésion avec la perception immédiate de leur identité. La règle du consensus communautaire autour de l'Etat est à la fois approuvée par le sens commun et justifiée par de savants 
développements lijphartiens à propos de la conversion du pluralisme ethnique en un pluralisme politique à travers un système d'équilibre entre minorités ${ }^{17}$. Certes, avec de nouvelles règles, le Liban de l'après-guerre joue un jeu ancien qui suscite toujours autant la perplexité. Sans revenir sur les critiques que nous avons formulées à propos $\mathrm{du}$ consensus entre élites communautaires dans le Liban de l'avant-guerre ${ }^{18}$, nous voudrions examiner deux caractéristiques saillantes de l'après-guerre: la contamination des pratiques politiques par l'esprit milicien, et la confusion entre intérêts publics et intérêts privés. Dans quelle mesure ces phénomènes renouvellent-ils le système communautaire? Et quels effets ont-ils sur la reconstruction de l'Etat libanais? Nous avons noté que la Loi constitutionnelle de 1990 reprenait, en le renforçant, le mode de représentation et de partage communautaire au sommet du pouvoir. Sa mise en application, à partir de la constitution du gouvernement d'Omar Karamé, le premier gouvernement de l'après-guerre, le 24 décembre 1990, montre un changement significatif dans l'identité des dirigeants du pays. Il ne s'agit pas seulement d'un changement générationnel après quinze ans de guerre, dont le très jeune ministre " Slimy " Frangié, petit-fils du Président Sleimane, est la figure emblématique. Il ne s'agit pas seulement non plus de l'entrée dans la classe politique de représentants des couches moyennes urbaines, comme le médecin Oussama Fakhoury ou l'avocat Joseph Moughaïzel. Il s'agit surtout de la cooptation de dirigeants d'organisations miliciennes, d'abord au gouvernement, ensuite au Parlement avec la nomination de 40 députés le 10 mai $1991^{19}$, à nouveau au Parlement par le moyen de l'élection sous surveillance de 128 députés dans l'été 1992, enfin dans l'administration lors de la nomination de nouveaux titulaires des cent vingt postes de fonctionnaires de première catégorie au printemps 1993 et au début de 1994. Aux côtés de Nabih Berri, de Walid Junblat, d'Elie Hobeiqa et, brièvement, de Samir Geagea, anciens chefs des trois grandes milices communautaires Amal (chiite), le PSP (druze) et les Forces Libanaises (maronite) - on trouve aussi des représentants des Maradas de Zghorta, du PSNS " grand syrien ", de la milice alaouite de Tripoli, le chef de l'Organisation populaire nassérienne de Saïda, un dirigeant des Ahbash - les Islamistes sunnites de Beyrouth - et, bien sûr, des responsables du Hizb Allah, seule milice à n'avoir pas été désarmée. La Loi d'amnistie 84 du 29 mars 1991, généreuse mais à l'application discrétionnaire, permet à tout ce beau monde de parader en habits civils dans les allées du pouvoir. En même temps, elle interdit tout travail collectif sur la mémoire de guerre à partir duquel une citoyenneté libanaise pourrait être reconstruite et, comme le fait remarquer l'ancien Premier ministre et député Salim el-Hoss, " pénalise ceux qui ont refusé de porter les armes ". Ces miliciens entrés en politique ont une conception de la res publica différente de celle des zu'âma traditionnels. D'une part, leur objectif n'est pas de dégager un consensus des élites, mais de faire triompher les intérêts de leur 'asabiyya. Walid Junblat, par exemple, réclame en 1991 la création d'un " ministère de la Montagne ", dont il escompte des bénéfices pour ses administrés druzes, comparables à ceux que Nabih Berri a tirés du " ministère du Sud " (créé en 1984) pour les Chiites, et surtout pour le mouvement Amal. Il obtiendra le ministère des Déplacés dans le gouvernement Hariri du 30 octobre 1992, autrement dit le contrôle d'une partie des subventions publiques et privées à la réinstallation des Chrétiens dans leurs villages du Chouf. D'autre part, ces ex-chefs miliciens n'ont guère de compétences techniques ou civiles, et la présence de certains d'entre eux dans les hautes sphères de l'équipement, de l'électricité, et même de la culture, est tout simplement atterrante. L'octroi d'une sinécure dans la fonction publique récompense une appartenance partisane, voire un fait d'armes; il est le 
résultat d'âpres marchandages au sein de la troïka des Présidents - Elias Hraoui, Nabih Berri et Rafiq Hariri - chacun cherchant à placer ses hommes et à récompenser sa clientèle plus qu'à assurer la gestion de l'Etat. La listes des " nominés " aux grandes directions de l'administration en mai 1993, reflète, outre les équilibres communautaires, le poids respectif des milices au sein de l'Etat. Elle mêle le nom de quelques professionnels compétents et intègres à ceux d'anciens chefs de gangs ${ }^{20}$ et autres apparatchiks, qui ont d'ailleurs plus d'affinités entre eux qu'avec les leaders traditionnels de leur communauté respective. Au sommet de l'Etat, les interminables négociations sur le mode mondain ont donc cédé la place aux pressions à peine voilées, aux déclarations agressives, aux jeux à somme nulle et aux exhibitions d'armes, encore innombrables après la mise en oeuvre de la Loi sur le désarmement des milices (23 mars 1991). " Chacun est le concurrent potentiel de tout le monde, aucune instance n'étant plus en mesure, ou en charge, d'empêcher le jeu et les surenchères de s'emballer à tout propos et hors de propos. L'affaiblissement du cran d'arrêt présidentiel a livré la vie politique et l'Etat aux démons de la dissension, de la volte-face, et de l'instabilité. Le rapport des forces, au sens brut et militaire de l'expression, qui ne devait servir que de donnée première transcendée au plus vite... s'avère une donnée permanente de la vie politique " ${ }^{21}$.

6 En quête de légitimité Transcender les antagonismes communautaires et substituer à la " culture de la discorde ${ }^{22}$ une culture commune (sinon nationale) est à la fois la raison d'être et le rôle d'un Etat. Encore faudrait-il que celui-ci soit porteur d'un projet hégémonique, sinon consensuel. Lors de la conquête militaire française en 1920, la compétition entre le Patriarcat maronite, promoteur d'un Liban chrétien, et les nouvelles élites modernisatrices qui oeuvraient au développement d'une culture politique transcommunautaire dans la mutasarifiyya (préfecture) ottomane, avait été tranchée en faveur du premier ${ }^{23}$. La raison d'être de l'Etat du grand Liban résidait bien moins dans la recherche d'un équilibre communautaire que dans la place centrale qu'il attribuait aux Chrétiens (aux Maronites en particulier, qui représentent à eux seuls la moitié des Chrétiens). Dans sa conception de l'espace (annexer à la Montagne la côte avec ses ports et la plaine intérieure avec ses terres céréalières), de l'identité (porter la marque du christianisme alors que tous les Etats arabes sont référés à l'islam), et de la géopolitique (se tourner vers l'Occident), le Liban devait être une " patrie pour les chrétiens ", même si y vivaient un grand nombre (et, bientôt, une majorité) de musulmans. L'élaboration juridique (article 17 de la Constitution de 1926) et surtout la pratique constitutionnelle d'un régime qui tendait peu à peu vers le présidentialisme confirmèrent l'hégémonie des Maronites auxquels la règle coutumière du Pacte national (1943) réserva la Présidence. Dès lors, la recherche d'un consensus et l'élaboration d'une politique nationale s'étaient construites pour et contre, au coeur de et autour de, cette prééminence chrétienne; pour et contre cette entité étatique spécifique dans son environnement proche-oriental. L'Etat communautaire de l'aprèsguerre se caractérise au contraire à la fois par l'absence d'une autorité supérieure et par le refus d'une prééminence communautaire : le pouvoir exécutif est diffusé entre les trois Présidences et plus encore à l'intérieur du Conseil des ministres ${ }^{24}$. Aucun groupe n'est plus en mesure de s'imposer aux autres, sous peine d'éclatement de la structure collective, mais aucune autorité libanaise n'est plus non plus en mesure de réguler la compétition. La guerre des communiqués, les démissions données, suspendues et reprises, et l'impéritie collective du cabinet Hariri face aux tâches de la reconstruction pour lesquelles il avait été constitué, en sont l'illustration flagrante. En 
fait, cet Etat se caractérise par l'absence de projet consensuel : plutôt que le vouloir vivre ensemble qui fonde la démocratie de consensus helvétique ou néerlandaise, les communautés libanaises partagent un devoir vivre ensemble, que ne vient étayer ni utopie ni idéologie ${ }^{25}$. C'est pourquoi le " désenchantement " chrétien signale une crise beaucoup plus profonde que la frustration de leaders qui ont perdu leurs positions de pouvoir, frustration que ne vient tempérer aucun partage de bénéfices économiques. Il marque la crise de perte de sens de l'Etat; son incapacité à coopter ceux qui restent en dehors du système et considèrent les autres communautés comme des rivaux, non des partenaires. Même si les opposants chrétiens à la nouvelle République ${ }^{26}$, les Aounistes, les ex-Forces Libanaises et leurs alliés, n'ont pas les moyens, et plus les ressources extérieures, de se soulever ou de faire sécession, leur refus d'y participer met en danger l'Etat communautaire, en ruinant sa précaire légitimité. Par contre, il y a bien un Etat qui tire avantage de l'insertion de la conflictualité au coeur du système politique libanais. Une puissance extérieure qui pallie l'absence de consensus interne par l'imposition d'une hégémonie externe armée. C'est la Syrie, dont les manoeuvres et la violence tout au long de la guerre lui ont assuré une position d'arbitre ${ }^{27}$. Il n'est pas une communauté, pas une milice au Liban, qui n'ait fait l'objet de la " contre-stratégie ethnique " ${ }^{28}$ de Hafez el-Assad durant quinze ans. Depuis Taëf, qui constituait une première victoire institutionnelle pour la Syrie ${ }^{29}$, cette politique a été confirmée et renforcée par l'Accord de fraternité et de coopération de mai 1991, puis par la tenue de sommets bilatéraux, la mise en place d'un Conseil supérieur mixte, et la signature de nombreux accords culturels et économiques. L'instrumentalisation du système social segmentaire et la manipulation du système politique d'équilibre par un régime qui ne cesse par ailleurs d'insister sur sa propre laïcité et d'invoquer son respect de la souveraineté de son petit voisin, ont abouti à ce paradoxe : rendre aussi indispensable qu'elle est insupportable sa présence armée au Liban. Dans les populations, la mémoire est encore fraîche du précédent du retrait de l'armée israélienne entre 1983-1985, qui a levé dans son sillage une multiplicité de guerres communautaires particulièrement meurtrières. Mais, plus anciennement, la précarité des trêves, la défaillance des entrepreneurs politiques et le recours à l'arbitrage étranger ne furent-ils pas les signes récurrents de la carence congénitale du système communautaire? La " longue paix " (1861-1915) dans la montagne libanaise aurait-elle duré sans le poids de l'administration ottomane ? La cité marchande aurait-elle prospéré, sans la férule du mandataire français qui tranchait dans les querelles locales? Par choix, mais aussi malgré lui, l'Etat libanais est devenu le grand absent de la vie politique du pays. Sans compter que, par le double jeu de la clôture communautaire et de l'arbitraire du pouvoir syrien, son interlocuteur indispensable, le citoyen, est entravé dans son épanouissement ${ }^{30}$.

7 Intérêts privés et intérêt public Le triomphe du néo-communautarisme milicien ne fait pas qu'exacerber la question de la légitimité de l'Etat. Il met aussi en cause sa fonction régulatrice, quand celle-ci devient plus nécessaire. Sans doute cette affirmation nécessiterait-elle d'être nuancée à un moment où la privatisation paraît à beaucoup un remède à la pénurie et à l'incurie de services publics comme le téléphone ou la distribution d'électricité; à un moment, aussi, où le Rassemblement des chefs d'entreprises libanais critique, dans son Livre blanc, la " place démesurée " de l'intervention étatique dans l'économie libanaise depuis $1975{ }^{31}$. Pourtant, les bases de la prospérité libanaise et les conditions de la reconstruction du pays ont profondément changé en l'espace des quinze années de guerre, nonobstant le triomphe de la doctrine 
néo-libérale et la mondialisation économique. D'abord, à cause des évolutions régionales: les Libanais ne retrouvent plus les positions lucratives qu'ils occupaient dans les Etats du Golfe, aujourd'hui plus développés et moins riches. Beyrouth n'est plus le pôle incontournable des échanges marchands, financiers et technologiques entre l'Orient arabe et l'Occident européen. A une économie de rente indirecte et de circulation, le Liban doit désormais substituer une économie de production, renouant avec un mouvement amorcé à la veille de la guerre. La définition d'un espace national, le développement d'un marché, l'attention au consommateur, sont les nouvelles exigences de l'heure. Ensuite, de nouveaux problèmes ont surgi dans les années 1990, d'ordre écologique, par exemple, mais surtout l'adaptation de l'économie libanaise aux exigences de la paix israélo-arabe. Y faire face relève de la responsabilité de la puissance politique plus que de celle des acteurs économiques. De même, les destructions de la guerre ont engendré des besoins spécifiques, par exemple en matière de logement, de formation et de réinsertion, autant de domaines qui relèvent de la logique sociale plus que de la logique économique. Enfin, la crise économique et financière, apparue plus tard que la crise politique et militaire, et qui se prolonge après la cessation des hostilités, est principalement une crise de confiance. Toutes ces nouvelles conditions réclament l'affirmation de l'acteur étatique et posent la question de sa capacité à défendre l'intérêt public. Avant la guerre, le communautarisme était source de distorsions dans le fonctionnement économique du Liban, en particulier parce que la répartition des postes de responsabilité se pliait au jeu des équilibres entre les différentes confessions, presqu'autant dans le secteur privé que dans le public. Mais, jusqu'en 1987, le capitalisme ultra-libéral a résisté au processus de fragmentation et de cloisonnement induit par la guerre et les affrontements miliciens. Il a maintenu une coopération transcommunautaire, en particulier dans le domaine bancaire, et facilité la circulation des personnes et des marchandises. Dans ce sens, il opérait comme substitut $\mathrm{du}$ système politique en crise, pour conserver au pays un minimum d'unité ${ }^{32}$. Au contraire, la stratégie des milices communautaires a été de prendre le contrôle des infrastructures et des ressources publiques sur chacun de leur territoire et de générer des économies autocentrées, tout en organisant conjointement les échanges aux frontières de ces territoires. En quinze ans de guerre, la recomposition de l'économie autour de pôles miliciens a transformé les paysages, avec la régionalisation et la croissance des villes secondaires, et modifié l'origine et la répartition des ressources : selon les estimations du Conseil du Développement et de la Reconstruction, dans la seconde moitié des années 1980, environ un tiers des revenus du Liban dépendait du contrôle des milices. L'économie de guerre était une économie de rente: salaires des miliciens et allocations aux familles de militants, aides d'une émigration extrêmement polarisée, ou de pays " protecteurs ", profits du commerce des armes et de la drogue, spéculation foncière, immobilière et monétaire, détournement des ressources publiques. C'était aussi une économie dispendieuse, puisqu'elle tendait à doubler, tripler ou plus, les infrastructures publiques (ports, raffineries, administration centrale, voire aéroports) sur un territoire grand comme le département de la Gironde ; une économie socialement déstabilisante, puisque les salariés des secteurs civils et de la fonction publique se prolétarisaient, tandis que s'enrichissaient les seigneurs de guerre et les entrepreneurs liés aux milices. Ce fonctionnement s'est prolongé après l'interruption des combats et le rétablissement de la légalité étatique. Pour ce faire, il s'est adapté à certaines exigences de la normalisation. En particulier, la culture du pavot, son trafic, et le commerce des armes sont rentrés dans la clandestinité, et pour 
l'instant marginalisés ${ }^{33}$. En l'espace de quelques mois, en 1991, les compagnies de transport, les coopératives d'approvisionnement, l'enlèvement des ordures, la sécurité routière, le cadastre.. tous ces services de substitution dépendant des milices ont fermé. Les milices ont aussi perdu le contrôle d'institutions publiques comme l'aéroport (Amal) ou le port de Beyrouth (le Vème bassin tenu par les FL), et surtout les ressources parafiscales qu'elles prélevaient en se substituant à l'Etat. Pour certaines institutions comme le Comité des travaux publics du PSP druze ou la Fondation de solidarité sociale, dirigée par un proche de Samir Geagea, la séparation forcée d'avec la milice a été l'occasion d'une reconversion vers des activités moins marquées confessionnellement : la défense civile, l'écologie, la protection des consommateurs ou l'aide aux handicapés. En même temps, des pans entiers de l'activité milicienne se sont abrités derrière des sociétés écrans, telle cette société d'ingénierie de Jounieh qui a recruté l'ensemble des ingénieurs militaires " démobilisés " par les FL. Ou bien les milices ont inscrit leurs entreprises au nom d'opérateurs privés, créé des sociétés de holding avec titres au porteur, et pris des participations croisées dans des sociétés basées à l'étranger. Mais le fonctionnement milicien n'a pas fait que s'effacer ou s'adapter au retour de la légalité civile. Il s'est aussi transporté au coeur de l'Etat par le truchement des chefs communautaires rentrés dans la légalité, induisant des conduites et impliquant des choix économiques lourds de conséquences. Il faut ici distinguer les pratiques clientélistes qui étaient celles des chefs communautaires d'avant-guerre ${ }^{34}$, du modèle qui préside à la reconstruction du Liban aujourd'hui, lequel s'apparente plutôt à un système prébendier largement extraverti ${ }^{35}$. En effet, le marché de la reconstruction publique et privée est potentiellement énorme - il a été évalué à 20 à 30 milliards de dollars pour un pays de 3 millions d'habitants. Les investisseurs et les entrepreneurs privés s'y taillent la part du lion, comme en témoigne l'opération emblématique de reconstruction du centre-ville de Beyrouth: malgré les vives polémiques qui ont accompagné les étapes de sa mise en chantier, les souscriptions auprès du promoteur, Solidere, ont dépassé en quelques semaines le montant requis ${ }^{36}$. Il n'y aurait là que logique libérale, ou même ultra-libérale, si l'Etat n'était pas un maillon essentiel du processus à la fois comme commanditaire principal des travaux, comme emprunteur sur le marché financier local, régional et auprès des organismes multilatéraux, et même comme producteur du cadre réglementaire dans lequel se concrétisent les opérations. Or, la présence au gouvernement et dans les hautes sphères de la fonction publique de personnalités représentant explicitement une communauté ou un segment communautaire, et tout aussi explicitement en compétition avec d'autres représentants d'autres segments communautaires, transforme inévitablement la recherche du bien public en défense des intérêts privés. Que ce soit la construction d'un hôpital dans la banlieue sud déshéritée de Beyrouth, la réunification de l'Université libanaise sur un vaste campus moderne, ou la récupération par la marine nationale du port militaire de Jounieh, l'intérêt " national " n'est que le paravent d'un enjeu implicite qui se situe, lui, au niveau communautaire. Les solution retenues, indemnisation exorbitante des squatters dans le premier cas, facultés dispersées que les étudiants mettront plusieurs heures à rejoindre dans le second, exceptionnalité de la mise en oeuvre d'un règlement qui épargne d'autres ports dans le troisième, sont les fruits du rapport de forces intercommunautaire. Lorsque l'Etat décide d'allouer une allocation de reconstruction aux villages du Jabal Amel après les bombardements israéliens de juillet 1993, lorqu'il subventionne la réinstallation des Chrétiens du Chouf dans les villages qu'ils avaient fuis en 1983, ce sont d'abord une structure 
communautaire et un leader politique qui en tirent les bénéfices financiers et symboliques. En retour, quand l'Etat, à la recherche de financements, appelle les expatriés à investir dans le pays, les puissantes associations d'émigrés organisées autour de pôles communautaires se querellent et choisissent des stratégies divergentes, dictées d'un côté par leur intérêt pour des régions spécifiques du pays, et de l'autre par les avantages qu'elles attendent de leurs coreligionnaires dans les rangs du pouvoir. Au delà de la reconstruction difficile du Liban, ce sont ses équilibres structurels qui sont mis en danger par des intérêts privés communautaires.

Etat ou système politique ? Sans sous-estimer les bénéfices de la fin de la guerre, et en particulier le retour de la sécurité sur les neuf dixièmes du pays, sans ignorer les progrès accomplis depuis quatre ans, il faut pourtant nous demander quel type d'Etat ont espéré rétablir les promoteurs de l'accord de Taëf, et quelle forme politique est entrain de se dessiner dans le Liban d'aujourd'hui. Entre exigence de déconfessionnalisation et calcification $\mathrm{du}$ communautarisme, entre besoins d'équipements collectifs et logiques des réseaux communautaires, leur choix met en cause la redéfinition même du pays, de sa place dans le Moyen-Orient du processus de paix, des modalités de représentation de ses populations, et de légitimation de son pouvoir politique. A chaque étape de notre examen du fonctionnement de la Deuxième république, nous avons observé que le processus de reconstruction est mené sous deux contraintes impératives, qui sont aussi deux tabous. D'abord, la contrainte des identités communautaires renforcées et des mobilisations centrifuges. Propriété de la terre, scolarisation des enfants, projets d'entreprises, l'esprit localiste s'impose, l'habitude de développement séparé reste prégnante et les frontières imaginaires puissantes. Les populations ne sont pas prêtes non plus à se plier aux contraintes d'un Etat unitaire (paiement des services publics et de l'impôt, conscription, arbitrage entre les priorités..), même si elles affichent une forte demande d'Etat, parce qu'elles ont souffert de l'oppression milicienne. L'Etat, pour sa part, persiste à nier les aspirations centrifuges. Il tergiverse devant la simple perspective d'une décentralisation administrative, pourtant inscrite au programme de Taëf. Du coup, il accentue la frustration des Druzes qui ambitionnent la constitution de deux départements druzes (un pour Rachaya et Hasbaya ; un pour le Chouf, Aley et le Haut Metn), et surtout celle des Chrétiens de l'opposition, qui réclament depuis longtemps une décentralisation politique. En faisant de la reconstruction du Centre ville sa priorité, il abandonne l'immense banlieue sud avec les problèmes aigus de logement, de circulation, d'approvisionnement en eau... d'un demi-million de Chiites, à la gestion de l'appareil milicien du Hizb Allah. Ainsi la négation du communautarisme s'accompagne-t-elle de pratiques communautaires renforcées. Ensuite, la contrainte de l'hégémonie syrienne : les intérêts et le mode d'exercice de la puissance syrienne vont à l'encontre de la reconstruction d'un espace public autonome libanais et d'une conscience citoyenne libanaise. Aussi longtemps que les négociations syro-israéliennes n'auront pas abouti, cette hypothèque pèsera sur le Liban. Le traitement communautaire du Liban, illustré par l'extension des réseaux économiques et politiques des barons du régime baassiste, attise la compétition locale entre les chefs communautaires en les vassalisant. Quand la normalisation adviendra au Proche-Orient, il pourrait être trop tard pour l'Etat libanais, surtout si son environnement régional (Israël et l'Arabie Saoudite) et international (les Etats-Unis et la France) néglige de lui fournir les appuis dont il aura besoin pour recouvrer sa souveraineté. Entre ces deux écueils, la renaissance de l'Etat libanais apparaît pour le moins problématique. Encore avons-nous laissé de côté la 
question de la faiblesse de la société civile ${ }^{37}$ et celle, non moins épineuse, de l'identité libanaise ${ }^{38}$. Peut-être, après avoir constaté le renouvellement du communautarisme et son nouveau mode de fonctionnement, et à la lumière des tensions et des guerres qui déchirent les régions pluri-communautaires d'Europe du Sud et du Caucase, devrionsnous remettre en cause la qualification d'Etat, s'agissant de l'entité libanaise, et lui substituer celle de système politique. Mais ce serait insulter l'avenir.

\section{NOTES}

1. Cette liste non exhaustive a été relevée dans l'article de Joseph Maïla, "le Document d'entente nationale, un commentaire", Les Cahiers de l'Orient, $n^{\circ}$ 16-17, 4ème trimestre 1989 - 1er trimestre 1990, pp. 135-217.

2. Qui marque la fin de la guerre civile, avec la chute du Général Aoun, le 13 octobre, et l'accord inter-chiite de Damas, le 6 novembre. La guerre régionale, israélo-arabe, et singulièrement israélo-syrienne, sur le territoire libanais s'est prolongée bien au-delà. 3. Interview de Paul Blanc, ancien ambassadeur de France à Beyrouth, à Al Mouaten, le Liban du Citoyen, 13, février 1993, p. 6.

4. L'expression est d'Ahmad Beydoun, Identité confessionnelle et temps social chez les historiens libanais contemporains, Beyrouth, Librairie orientale, 1984, p. 333.

5. Lors de la rencontre du CERI du 30 mai 1994 sur la crise de l'Etat au Liban.

6. Ce thème est développé dans les travaux de Riva Kastoryano sur les communautés dans l'émigration.

7. Al-Jumhûriyya al-lubnâniyya, Majlis al-Nuwwâb, Al-Dastûr al-lubnânî, Beyrouth, 1990, p. 78.

8. Onzième et dernier principe général du Document d'entente nationale. Parmi les réformes politiques exposées dans le titre 2, figure la distribution paritaire (entre chrétiens et musulmans) et proportionnelle (entre communautés) des sièges parlementaires.

9. Mot à mot "celles qui agissent". Ce terme désigne les nouvelles élites issues de la guerre.

10. Document d'entente pour le Liban, titre II, l'Extension de la souveraineté de l'Etat libanais sur la totalité du territoire libanais, paragraphe de conclusion : "les forces syriennes... aideront les forces libanaises à étendre l'autorité de l'Etat au cours d'une période maximale de deux ans commençant après... l'adoption constitutionnelle des réformes politiques". Cahiers de l'Orient, $\mathrm{N}^{\circ} 16-17,4 \mathrm{e}$ trim. 89 - 1er trim. 90, p. 127. 11. Sur cette transformation, voir Georges Corm, Contribution à l'étude des sociétés multi-confessionnelles, effets socio-politiques et juridiques du pluralisme religieux, Paris, LGDJ 1971, Appendice III, pp. 273-287.

12. Par un processus démonté par Michel Seurat, l'Etat de Barbarie, Paris, Le Seuil, 1989. Les militaires baassistes alaouites de Syrie utilisent leur 'asabiyya, leurs liens de solidarité communautaire, pour accaparer le pouvoir. Au nom de l'intégration nationale, ils interdisent aux autres communautés d'en faire autant. 
13. Sur la difficulté de penser la démocratie dans des Etats où la nation reste à inventer, voir Ghassan Salamé, "Où sont les démocrates ?", pp. 16-25 in Démocraties sans démocrates. Politiques d'ouverture dans le monde arabe et islamique, Ghassan Salamé (dir.) Paris, Fayard, 1994.

14. Voir sa contribution sur le "Système des milices" in Peace for Lebanon : from war to reconstruction, Deirdre Collings (dir.), Boulder, Lynne Rienner, 1994.

15. Voir Joseph Bahout, "Liban : les élections législatives de l'été 1992", MaghrebMachrek, 139 (janvier-mars 1993), pp. 53-84.

16. Au début des années 1970, des intellectuels maronites réclamaient la "sécularisation de l'Etat", convaincus que leur communauté assurerait son hégémonie, non par la loi du nombre - les Maronites n'étaient déjà plus la communauté la plus nombreuse - mais en vertu de leur niveau de compétence. Voir le Memorandum du parti Kataëb au président de la République et au Patriarche maronite, al-Nahâr 24 août 1975, p. 2 \& 12. 17. Les nombreux travaux du politologue Antoine Messara sont inspirés des travaux d'Arendt Lijphardt sur la démocratie de consensus. Voir en particulier Le modèle libanais et sa survie, Beyrouth, Librairie Orientale, 1983. C'est aussi la thèse qui soustend l'ouvrage, à notre sens peu convainquant, de Theodor Hanf, Coexistence in wartime Lebanon, Decline of a state and rise of a nation, Londres, I.B.Tauris, 1993. 18. Qui nous ont été inspirées par Arendt Lijphart lui-même, Democracy in plural societies. A comparative exploration, New Haven, Yale university press, 1977, p. 303. 19. Un député par "petite "milice, deux à trois par "grande" milice (Amal, Parti Socialiste Progressiste et Forces Libanaises).

20. Voir Magazine, 4 juin 1993, p.19-20, pour le détail des postes et des appartenances communautaires et miliciennes.

21. Waddah Charara, "Deux ans de réunification nationale : une libanisation gigogne", Cahiers de la Méditerranée, 44, juin 1992, p. 168.

22. Voir Georges Corm, "Sur la culture de la discorde", Liban : les guerres de l'Europe et de l'Orient, 1940-1992, Paris, Gallimard Folio, 1992, pp. 349-431.

23. Sur la génèse d'une culture politique transcommunautaire dans la mutasarifiyya du Mont Liban, voir Engin Akarli, The long peace, Ottoman Lebanon 1861-1920, Berkeley, University of California press, 1993. Sur le choix du Grand Liban, qu'il considère comme une erreur, voir Meir Zamir, The formation of modern Lebanon, Londres, Croom Helm, 1985.

24. Al-Dastûr al-Lubnânî, op. cit., p. 51 sq.

25. Ghassan Salamé, "La démocratie comme instrument de paix civile", Démocraties sans démocrates, op. cit., p. 154.

26. Et qu'il faut bien distinguer des opposants au gouvernement, comme par exemple les membres du bloc de Salim al-Hoss ou même le Hizb Allah.

27. Voir Elizabeth Picard, "La politique de la Syrie au Liban. Les développements incontrôlables d'une stratégie ambitieuse", Maghreb-Machrek, 116, avril-juin 1987, pp. 5-34. Et d'excellents développements dans Samir Kassir, La guerre du Liban. De la dissension nationale au conflit régional, Paris, Karthala- CERMOC, 1994.

28. Cette formule est empruntée au texte introductif de Bertrand Badie et Christophe Jaffrelot.

29. Le Document d'entente consacre un long développement dans son titre II (Extension de la souveraineté de l'Etat) et tout le titre IV (les relations libanosyriennes) au rôle de la Syrie au Liban. 
30. Voir Nawaf Salam, "Individu et citoyen au Liban", pp.137-150 in Le Liban aujourd'hui, (sous la dir. de Fadia Kiwan), Paris, CERMOC-CNRS, 1994.

31. RDCL, Livre blanc de l'économie libanaise, cité par Fouad Khoury-Helou, "Le livre blanc de l'économie libanaise, Al Mouaten, 20, mai 1994, p. 12. L'économiste Kamal Hamdan relève que "l'appareil [administratif] est devenu de plus en plus lourd et coûteux et de moins en moins productif et efficace" puisque le budget de l'Etat représentait $40 \%$ et même $50 \%$ du PIB. Kamal Hamdan, "La classe moyenne dans la guerre du Liban", in Le Liban aujourd'hui, op. cit., p. 201.

32. Voir Clement Henry Moore, "Le système bancaire libanais. Les substituts financiers d'un ordre politique", Maghreb-Machrek, 99, janvier-mars 1983, pp. 30-46.

33. Voir Hassan Makhlouf, Culture et trafic de drogue au Liban, Paris, L'Harmattan, 1994.

34. Selon le modèle décrit par Michael Johnson, "Political bosses and their gangs : zu'ama and qabadayat in the Sunni muslim quarters of Beirut", in Patrons and clients in Mediterranean societies, Ernest Gellner \& John Waterbury (dir.), Londres, Duckworth, 1977, pp. 194-216.

35. Sur la prébende, et plus généralement sur la dynamique des relations entre position de pouvoir et accumulation, voir Jean-François Bayart, l'Etat en Afrique, Paris, Fayard, 1989, pp. 104-112.

36. 926 millions de dollars, alors qu'il en était offert 650 à la souscription. Voir Le Monde, 15 janvier 1994.

37. L'étude de Brigitte Curmi sur les ONG libanaises, in Ingénieurs et médecins dans le monde arabe, d'Elisabeth Longuenesse (dir.), à paraître (1994), montre que la majorité des organismes sociaux et médicaux développés depuis la guerre ont un caractère communautaire marqué.

38. Voir Roula Jammal Mouawiyah, L'identité socioculturelle libanaise et ses représentations. Etude d'une population immigrée à Paris, thèse de doctorat en psychologie sociale, Paris, EHESS, 1994. A travers une enquête fine et bien exploitée, Roula Mouawiyah montre la réalité d'une identité socioculturelle, face à une identité nationale chancelante.

INDEX

Mots-clés : démocratie, Etat-nation, Nationalismes

Index géographique : Liban

Thèmes : Hezbollah 